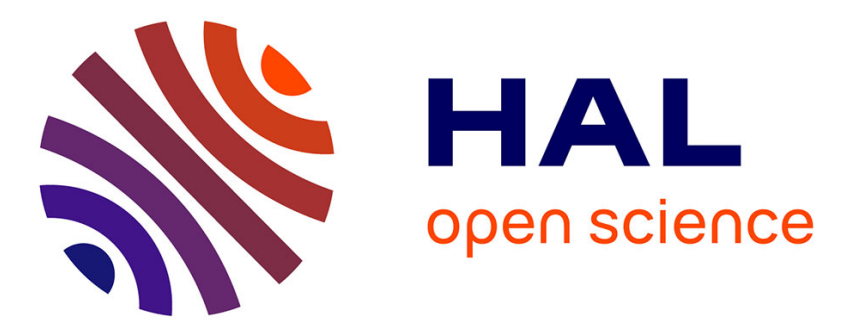

\title{
Motion planning for non-holonomic mobile robots using the i-PID controller and potential field
} Yingchong Ma, Gang Zheng, Wilfrid Perruquetti, Zhaopeng Qiu

\section{To cite this version:}

Yingchong Ma, Gang Zheng, Wilfrid Perruquetti, Zhaopeng Qiu. Motion planning for non-holonomic mobile robots using the i-PID controller and potential field. IEEE/RSJ Conference on Intelligent Robots and Systems (IROS), 2014, Sep 2014, Chicago, United States. hal-01071506

\section{HAL Id: hal-01071506 \\ https://inria.hal.science/hal-01071506}

Submitted on 30 Oct 2014

HAL is a multi-disciplinary open access archive for the deposit and dissemination of scientific research documents, whether they are published or not. The documents may come from teaching and research institutions in France or abroad, or from public or private research centers.
L'archive ouverte pluridisciplinaire HAL, est destinée au dépôt et à la diffusion de documents scientifiques de niveau recherche, publiés ou non, émanant des établissements d'enseignement et de recherche français ou étrangers, des laboratoires publics ou privés. 


\title{
Motion planning for non-holonomic mobile robots using the $i$-PID controller and potential field
}

\author{
Yingchong Ma, Gang Zheng, Wilfrid Perruquetti and Zhaopeng Qiu
}

\begin{abstract}
This paper proposes a motion planning approach for non-holonomic mobile robots. Firstly, motion planning using $i$-PID controller is presented. Then we improve the old potential field function to produce smooth repulsive force. Finally a new repulsive function of robot orientation and angular velocity is proposed to improve the performance of obstacle avoidance. The effectiveness and the robustness of the proposed method are shown thereafter via several simulations.
\end{abstract}

\section{INTRODUCTION}

Robot motion planning has been studied for many years, and there are many different studies using different approaches for motion planning problems [1], such as $A^{*}$ [2], $D^{*}$ [3], artificial potential field [4], visibility graph [5] and methods based on optimal control problem [6].

Artificial potential field is proposed by Khatib [7] and has been widely studied because of its simplicity and interesting mathematical analysis [8], [9]. The basic idea of this approach is to fill the robot workspace with attractive potential field caused by the target and the repulsive potential field caused by obstacles. There are also some extensions for the potential field method. Moving obstacles are considered in [10]. In [11] the velocity of obstacles and target are taken into consideration, and the unreachable target with obstacle nearby problem is discussed in [12]. Although the basic idea of potential field approach seems easy, there exist some situations that the avoidance of obstacles can not be achieved, and oscillatory movement is also an important issue.

As for non-holonomic mobile robots, the design of feasible trajectory joining the starting position and the target is not straightforward because of the non-holonomic constraint. Both open-loop and close-loop methods are designed [13]. However open-loop methods are not robust to the disturbance in the system. Closed-loop methods of non-holonomic mobile robots have been studied, but according to Brockett's theorem [14], there exists no smooth feedback control that stabilizes the given configuration. This means that the class of the controllers should be extended to take into account time-varying or non-smooth controllers [15].

\footnotetext{
Yingchong Ma, Zhaopeng Qiu and Wilfrid Perruquetti are with LAGIS CNRS UMR 8219, Ecole Centrale de Lille, BP 48, 59651 Villeneuve d'Ascq, France \{yingchong.ma, zhaopeng.qiu\}@ec-lille.fr

Wilfrid Perruquetti and Gang Zheng are with Non-A team, INRIA Lille Nord Europe, 40 avenue Halley, 59650 Villeneuve d'Ascq, France \{wilfrid.perruquetti, gang.zheng\}@inria.fr

This work was supported by EU INTERREG IVA 2 Mers Seas Zeeen Cross-border Cooperation Programme under SYSIASS project 06-020. It was also supported by Ministry of Higher Education and Research, NordPas de Calais Regional Council and FEDER through the "Contrat de Projets Etat Region (CPER) CIA 2007-2013”.
}

In this paper, we propose to use the robust $i$-PID controller for robot motion planning. The desired direction is generated by a new potential field function to improve the performance of obstacle avoidance. The new potential function is defined by taking into account the robot orientation and angular velocity besides the position and linear velocity, and distance criteria is added to the original potential function to decrease oscillations. This method only needs the online measurements of the robot velocity and information of obstacles.

The outline of this paper is as follows. Problem statement is given in section II. Robot motion planning using $i$-PID controller is described in section III. The new potential functions are defined in section IV. Simulation results are detailed in section V.

\section{PROBLEM STATEMENT}

Generally speaking, the total force generated by potential function can be used for controller design. A common way is to set a positive linear velocity for the robot and design an angular controller to make the robot orientation converge to the angle of the total force. While this method is not robust to the disturbances in the system, therefore the first contribution of this paper is to propose an robust $i$-PID controller to track the desired velocities.

In robot motion planning problems, the potential forces need to change smoothly to achieve good tracking performance. Moreover the original potential field approach has some drawbacks. In Fig. 1, the direction of the total force $\mathbf{S}_{\text {total }}{ }^{1}$ imposed on the robot pass through the obstacle center, thus the robot can not move away from the obstacle. In [11] another force is introduced in the direction of the velocity. However as seen in Fig. 2, when the direction of the velocity passes through the obstacle center, the robot can not avoid the obstacle. This motivates us to think whether we can introduce a force in the direction perpendicular to the robot direction when the robot is approaching the obstacle. Therefore we take the orientation and the angular velocity of the robot into consideration to propose a new potential function, which is the second contribution of this paper.

\section{MOTION PLANNING FOR NON-HOLONOMIC MOBILE ROBOTS VIA $i$-PID CONTROLLER}

\section{A. Problem formulation}

The objective of motion planning for mobile robots is to control the robot following the direction of a total force $\theta_{s}$ generated by the potential functions.

\footnotetext{
${ }^{1}$ The boldface type is used for vectors in this paper.
} 


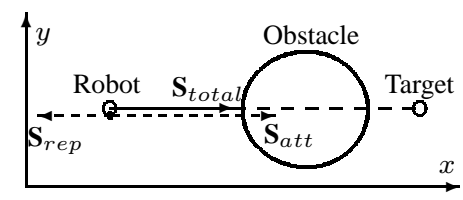

Fig. 1. Drawbacks in original potential field method based on robot position

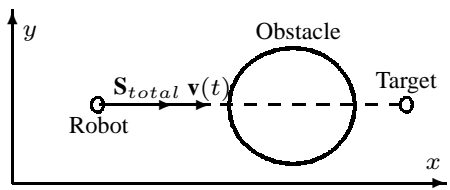

Fig. 2. Drawbacks in potential field method based on robot position and linear velocity

In this paper, the $i$-PID controller is used since it is robust to the disturbances and noises in the system. We regard the direction following problem as a desired velocity tracking problem, since $\frac{v_{r, y}}{v_{r, x}}=\frac{\mathbf{S}_{y}}{\mathbf{S}_{x}}=\tan \theta_{s}$, where $v_{r, x}$ and $v_{r, y}$ are desired velocities on $x$ and $y$ axis respectively, and $\mathbf{S}_{x}$ and $\mathbf{S}_{y}$ are the component of the total force $\mathbf{S}_{\text {total }}$ on $x$ and $y$ axis respectively. Thus, if one can make the robot velocities on $x$ (which is equal to $\dot{x}$ ) and $y$ (which is equal to $\dot{y}$ ) to track $v_{r, x}$ and $v_{r, y}$ respectively, then one can ensure the robot moving with the desired angle $\theta_{s}$.

In fact, this new interpretation by tracking desired velocity has an important advantage: it can take into account some physical constraints of robots, such as maximal velocity. For example, since $v_{r, y}=\frac{\mathbf{S}_{y}}{\mathbf{S}_{x}} v_{r, x}=K_{s} v_{r, x}$, where $K_{s}=\frac{\mathbf{S}_{y}}{\mathbf{S}_{x}}$ is known and deduced from potential field function. If $K_{s} \geq 1$, one can set the desired velocities as $v_{r, y}=v_{m y}, v_{r, x}=\frac{\bar{v}_{m y}}{K_{s}}$, where $v_{m y}=v_{\max } \sqrt{\frac{K_{s}^{2}}{1+K_{s}^{2}}}$. If $K_{s}<1$, one can define the desired velocities as $v_{r, x}=v_{m x}, v_{r, y}=K_{s} v_{m x}$, where $v_{m x}=\frac{v_{\max }}{\sqrt{1+K_{s}^{2}}}$. In both cases, we can always ensure that the physical constraints are satisfied. Then the velocity tracking problem is described in the following.

\section{B. Robot model}

Let us consider the unicycle-type mobile robot whose kinematic model under the non-holonomic constraint of pure rolling and no slipping can be described as follows:

$$
\left\{\begin{array}{l}
\dot{x}=v \cos \theta \\
\dot{y}=v \sin \theta \\
\dot{\theta}=\omega
\end{array}\right.
$$

where $v$ and $\omega$ are linear and angular velocity respectively, $x$ and $y$ represent the location of the robot, $\theta$ is the orientation of the robot with respect to $x$-axis.

In [16], a simple controller is obtained to track the desired velocities $v_{r, x}$ and $v_{r, y}$ by using pseudoinversion. From (1) we have

$$
\left[\begin{array}{l}
\dot{x} \\
\dot{y}
\end{array}\right]=G(\theta)\left[\begin{array}{l}
v \\
\omega
\end{array}\right]
$$

where $G(\theta)=\left[\begin{array}{cc}\cos \theta & 0 \\ \sin \theta & 0\end{array}\right]$, thus the controller proposed in
[16] is as follows:

$$
\left[\begin{array}{c}
v \\
\omega
\end{array}\right]=\left[G^{T} G\right]^{-1} G^{T}\left[\begin{array}{l}
v_{r, x} \\
v_{r, y}
\end{array}\right]
$$

However, if the initial condition of the robot is different with the reference, this approach can not converge the velocity to the reference, since the proposed controller in [16] cannot guarantee the asymptotical convergence: $\dot{x} \rightarrow v_{r, x}$ and $\dot{y} \rightarrow v_{r, y}$. In order to ensure the convergence, an intuitive solution is to consider the dynamics of $\ddot{x}$ and $\ddot{y}$, and design a controller to make $\dot{x}$ and $\dot{y}$ track the desired velocity $v_{r, x}$ and $v_{r, y}$.

For this, let us consider the following dynamics deduced from the system model (1):

$$
\left[\begin{array}{l}
\ddot{x} \\
\ddot{y}
\end{array}\right]=\tilde{G} \tilde{u}
$$

where

$$
\tilde{G}=\left[\begin{array}{cc}
\cos \theta & -v \sin \theta \\
\sin \theta & v \cos \theta
\end{array}\right]
$$

and $\tilde{u}=[\dot{v}, \omega]^{T}$ being the new control input. The following section is then devoted to using the robust $i$-PID controller to achieve the tracking task.

\section{C. $i$-PID controller}

The $i$-PID controller was proposed in [17], and it exhibits robustness to the unmodeled dynamics and disturbance in the system [18]. Let us briefly present the basic idea of this controller.

Generally speaking, this method locally approximates the system model by a local model with an unknown term, and the unknown term can be estimated by the measurements of the input and output of the system, then a so-called $i$-PID controller can be deduced to realize the control goal.

In this paper the system model (4) is approximated by the following local model over a small time interval $T=$ $\left[t_{k}, t_{k+1}\right]$, with $k \in Z^{+}$:

$$
\left[\begin{array}{l}
\ddot{x} \\
\ddot{y}
\end{array}\right]=F(t)+\alpha(x, y, \dot{x}, \dot{y}) \tilde{u}(t)
$$

where $\alpha(x, y, \dot{x}, \dot{y})$ is a non singular $2 \times 2$ dimensional matrix which should be well chosen in order to achieve the control goal. $F(t) \in \mathbb{R}^{2}$ represents all unknown terms including disturbances, which can be estimated by using the information of $\tilde{u}, \alpha(x, y, \dot{x}, \dot{y})$ and the output of the system.

For the above continuous local model over time $T$, one can estimate $F(t)$ by discretizing it. Denote $T_{s}$ as the sampling time period, so that at each step $k=t / T_{s}$ we have

$$
\left[\begin{array}{c}
\ddot{x}_{k} \\
\ddot{y}_{k}
\end{array}\right]=F_{k}+\alpha(x, y, \dot{x}, \dot{y}) \tilde{u}_{k}
$$

then it yields

$$
F_{k}=\left[\begin{array}{c}
\ddot{x}_{k} \\
\ddot{y}_{k}
\end{array}\right]-\alpha(x, y, \dot{x}, \dot{y}) \tilde{u}_{k}
$$

where $\left[\ddot{x}_{k}, \ddot{y}_{k}\right]^{T}$ and $\tilde{u}_{k}$ are measurable signals at time $k$. If it assumed that $T_{s}$ is small enough such that $F_{k-1} \rightarrow F_{k}$, then the $i$-PID controller can be designed as follows:

$$
\tilde{u}_{k}=\alpha^{-1}(x, y, \dot{x}, \dot{y})\left(F_{k-1}+e_{k}\right)
$$


where

$$
e_{k}=\left[\begin{array}{c}
\ddot{v}_{r, x} \\
\ddot{v}_{r, y}
\end{array}\right]+K_{p}\left[\begin{array}{c}
\dot{x}-v_{r, x} \\
\dot{y}-v_{r, y}
\end{array}\right]+K_{I} \int_{T}\left[\begin{array}{l}
\dot{x}-v_{r, x} \\
\dot{y}-v_{r, y}
\end{array}\right]
$$

and $K_{p}$ and $K_{I}$ are usual tuning gains. The determination of $\alpha(x, y, \dot{x}, \dot{y})$ and the calculation of $F_{k}$ are exhaustively discussed in [19]. Due to the limit of pages, we just recall that $F_{k}$ is calculated as

$$
F_{k}=\frac{6}{T} \int_{0}^{1}(2 \delta-1)\left[\begin{array}{c}
\dot{x} \\
\dot{y}
\end{array}\right] d \delta+6 \alpha(x, y, \dot{x}, \dot{y}) \int_{0}^{1}\left(\delta^{2}-\delta\right) \tilde{u} d \delta
$$

where $\delta \in[0,1]$. For more details on the determination of $\alpha$ and $F_{k}$, please refer to [19].

\section{Potential FiEld FUnCtion}

\section{A. Attractive potential function}

The reference for the motion controller used above is generated from potential field functions, let us firstly define the attractive potential field function. Like other potential field methods, it is defined as a function of relative distance between the robot and the target:

$$
U_{a t t}(\mathbf{P})=K_{a t t}\left\|\mathbf{P}_{t a r}-\mathbf{P}(t)\right\|^{2}
$$

where $\mathbf{P}(t)$ and $\mathbf{P}_{t a r}$ denote the positions of the robot and the target at time $t$ respectively, $\left\|\mathbf{P}_{\text {tar }}-\mathbf{P}(t)\right\|$ is the Euclidean distance between the robot and the target, and $K_{a t t}$ is a positive parameter.

Therefore the desired attractive force is defined as the negative gradient of the attractive potential function with respect to the robot position:

$$
\mathbf{S}_{a t t}(\mathbf{P})=-\nabla_{\mathbf{P}} U_{a t t}(\mathbf{P})=-\frac{\partial U_{a t t}(\mathbf{P})}{\partial \mathbf{P}}=-2 K_{a t t}\left\|\mathbf{P}_{t a r}-\mathbf{P}(t)\right\| \mathbf{n}_{R T}
$$

where $\mathbf{n}_{R T}$ is the unit vector pointing from the robot to the target.

\section{B. Repulsive potential function}

Conventionally, the repulsive function is defined as a function of relative distance between the robot and obstacles. In this paper a new repulsive function makes fully use of the position, velocity, orientation and angular velocity of the robot is presented to improve the performance of obstacle avoidance.

According to [11], the repulsive potential function of robot position and linear velocity is considered as follows. Define $P_{d}\left(\mathbf{P}, \mathbf{P}_{\text {obs }}\right)$ as the distance between the robot and an obstacle, and $V_{R O}(t)=\mathbf{v}(t)^{T} \mathbf{n}_{R O}$ as the velocity in the direction from the robot to the obstacle, where $\mathbf{v}(t)$ is the robot velocity and $\mathbf{n}_{R O}$ is a unit vector point from the robot to the obstacle. Define $a_{\max }$ is the maximum deceleration of the robot, thus the distance traveled by the robot before $V_{R O}$ reduces to 0 is $P_{m}\left(V_{R O}\right)=\frac{V_{R O}^{2}}{2 a_{\max }}$. Then the repulsive potential function is defined as

$$
\begin{aligned}
& U_{r e p}(\mathbf{P}, \mathbf{v})= \\
& \left\{\begin{array}{l}
0, \quad \text { if } P_{d}\left(\mathbf{P}, \mathbf{P}_{\text {obs }}\right)-P_{m} \geq p_{0} \text { or } V_{R O} \leq 0 \\
K_{p v}\left(\frac{1}{P_{d}\left(\mathbf{P}, \mathbf{P}_{o b s}\right)-P_{m}}-\frac{1}{p_{0}}\right), \\
\quad \text { if } 0<P_{d}\left(\mathbf{P}, \mathbf{P}_{\text {obs }}\right)-P_{m}<p_{0} \text { and } V_{R O}>0
\end{array}\right.
\end{aligned}
$$

where $p_{0}$ is the influence range of the obstacle, and $K_{p v}$ is a positive constant.

However one can notice in (14) that if $V_{R O}(t)$ changes from positive to negative in one step, this will introduce a sudden change in the value of repulsive potential function $U_{\text {rep }}(\mathbf{P}, \mathbf{v})$. When considering the repulsive force, i.e. the derivative of this repulsive potential, it will result in frequent oscillations in complex environment, which is not expected for controller design. To overcome this problem, we multiply the original repulsive potential function by $\cos \theta_{d}$, where $\theta_{d}=\left\|\theta-\theta_{R O}\right\|$, and $\theta$ is the orientation of the robot, $\theta_{R O}$ is the angle of the vector $\mathbf{n}_{R O}$. The new $U_{\text {rep }}(\mathbf{P}, \mathbf{v})$ is as follows:

$$
\begin{aligned}
& U_{r e p}(\mathbf{P}, \mathbf{v})= \\
& \left\{\begin{array}{l}
0, \quad \text { if } P_{d}\left(\mathbf{P}, \mathbf{P}_{o b s}\right)-P_{m} \geq p_{0} \text { or } V_{R O} \leq 0 \\
K_{p v}\left(\frac{1}{P_{d}\left(\mathbf{P}, \mathbf{P}_{o b s}\right)-P_{m}}-\frac{1}{p_{0}}\right) \cos \theta_{d}, \\
\text { if } 0<P_{d}\left(\mathbf{P}, \mathbf{P}_{o b s}\right)-P_{m}<p_{0} \text { and } V_{R O}>0
\end{array}\right.
\end{aligned}
$$

which guarantees the repulsive potential function reduce to zero smoothly while $V_{R O}(t)$ becomes negative. Moreover a new repulsive potential function based on robot orientation and angular velocity is presented in the following to improve the performance of obstacle avoidance.

Let us define that if a maximum deceleration $\beta_{\max }$ is applied to the robot to reduce its angular velocity, the angle traveled by the robot before angular velocity $w(t)$ reduces to zero is:

$$
\theta(\omega)=\frac{\omega(t)^{2}}{2 \beta_{\max }}
$$

Then the repulsive potential function of robot orientation and angular velocity is defined as follows:

$$
\begin{aligned}
& U_{r e p}(\theta, \omega)= \\
& \left\{\begin{array}{l}
0, \quad \text { if } V_{R O}<0 \text { or } P_{d}>p_{\theta} \text { or } \theta_{d}>\theta_{0} \\
\left(-K_{\theta 1}\left(\theta_{d}-\theta(\omega)\right)^{2}+K_{\theta 2}\right)^{2}\left(p_{\theta}-P_{d}\left(\mathbf{P}, \mathbf{P}_{o b s}\right)\right)^{2}\left(\theta_{0}-\theta_{d}\right)^{2}, \\
\quad \text { if } V_{R O}>0 \text { and } P_{d} \leq p_{\theta} \text { and } \theta(\omega)<\theta_{d} \leq \theta_{0} \\
K_{\theta 2}^{2}\left(p_{\theta}-P_{d}\left(\mathbf{P}, \mathbf{P}_{o b s}\right)\right)^{2}\left(\theta_{0}-\theta_{d}\right)^{2}, \\
\text { if } V_{R O}>0 \text { and } P_{d}<p_{\theta} \text { and } \theta_{d}<\theta(\omega)
\end{array}\right.
\end{aligned}
$$

where $p_{\theta}$ is a positive constant describing the influence range of obstacle on $U_{r e p}(\theta, \omega)$, and $p_{\theta}>p_{0}, \theta_{0}$ is the influence angle, $K_{\theta 1}$ and $K_{\theta 2}$ are positive constants. As shown in Fig. 3 , the direction of $\mathbf{v}_{1}(t)$ is out of the influence angle $\theta_{0}$, thus $U_{\text {rep }}(\theta, \omega)=0$. In the second case, the direction of $\mathbf{v}_{2}(t)$ is in the influence angle $\theta_{0}$ but out of the range of $\theta(\omega)$, the potential function is of paraboloidal profile. In the last case, the direction of $\mathbf{v}_{3}(t)$ is inside angle $\theta(\omega)$, the potential function is defined as the maximum value of the parabola.

One can notice that in (17), $\left(p_{\theta}-P_{d}\left(\mathbf{P}, \mathbf{P}_{\text {obs }}\right)\right)^{2}$ makes the value of $U_{\text {rep }}(\theta, \omega)$ change smoothly when robot reaches and leaves the influence range of the obstacle, and $\left(\theta_{0}-\theta_{d}\right)^{2}$ makes the value of $U_{r e p}(\theta, \omega)$ change smoothly when $\theta_{d}$ reaches and exceeds the influence angle. The smooth change of $U_{a t t}(\theta, \omega)$ is very beneficial for tracking.

The new repulsive potential function is defined as

$$
U_{r e p}=U_{r e p}(\mathbf{P}, \mathbf{v})+U_{r e p}(\theta, \omega)
$$




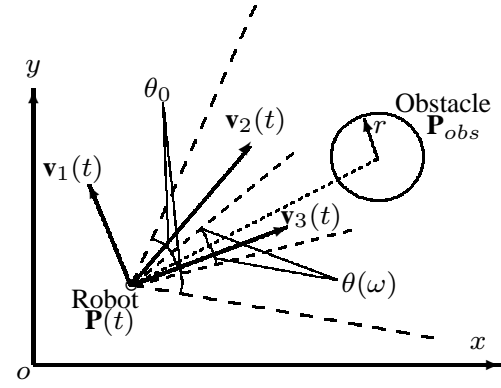

Fig. 3. Different cases of $U_{r e p}(\theta, \omega)$

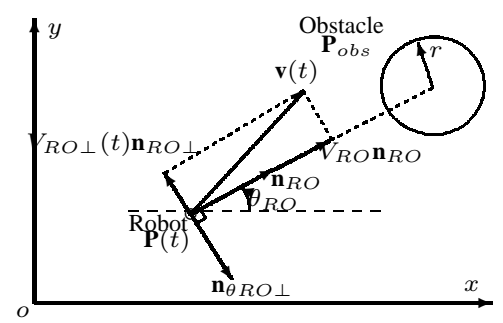

Fig. 4. Vectors for defining repulsive force

Thus the corresponding repulsive force $\mathbf{S}_{\text {rep }}$ is defined as the negative gradient of the repulsive potential function with respect to robot position and velocity. Since $\nabla_{\mathbf{v}} U(\theta, \omega)=0$, we have

$$
\begin{aligned}
\mathbf{S}_{r e p} & =\mathbf{S}_{r e p}(\mathbf{P}, \mathbf{v})+\mathbf{S}_{r e p}(\theta, \omega) \\
& =-\nabla_{\mathbf{P}} U_{r e p}(\mathbf{P}, \mathbf{v})-\nabla_{\mathbf{v}} U_{r e p}(\mathbf{P}, \mathbf{v})-\nabla_{\mathbf{P}} U_{r e p}(\theta, \omega)
\end{aligned}
$$

The robot velocity in the direction from the robot to the obstacle $V_{R O}$ can be written as

$$
\begin{aligned}
V_{R O}(t) & =\mathbf{v}(t)^{T} \mathbf{n}_{R O} \\
& =\mathbf{v}(t)^{T} \frac{\mathbf{P}_{o b s}-\mathbf{P}(t)}{\left\|\mathbf{P}_{o b s}-\mathbf{P}(t)\right\|}
\end{aligned}
$$

then the gradients of $V_{R O}(t)$ with respect to velocity and position are calculated as

$$
\begin{gathered}
\nabla_{\mathbf{v}} V_{R O}(t)=\mathbf{n}_{R O} \\
\nabla_{\mathbf{P}} V_{R O}(t)=\frac{V_{R O}(t) \mathbf{n}_{R O}-\mathbf{v}(t)}{\left\|\mathbf{P}_{o b s}-\mathbf{P}(t)\right\|}
\end{gathered}
$$

For clarity, as shown in Fig. 4, denote $V_{R O \perp}(t) \mathbf{n}_{R O \perp}$ as the velocity component perpendicular to $V_{R O}(t) \mathbf{n}_{R O}$, which is given in the following equation:

$$
V_{R O \perp}(t) \mathbf{n}_{R O \perp}=\mathbf{v}(t)-V_{R O}(t) \mathbf{n}_{R O}
$$

where

$$
\begin{aligned}
& V_{R O \perp}(t)=\sqrt{\mathbf{v}(t)^{2}-V_{R O}(t)^{2}} \\
& \mathbf{n}_{R O \perp}^{T} \mathbf{n}_{R O}=0
\end{aligned}
$$

thus equation (22) can be written as

$$
\nabla_{\mathbf{P}} V_{R O}(t)=\frac{-V_{R O \perp} \mathbf{n}_{R O \perp}}{\left\|\mathbf{P}_{o b s}-\mathbf{P}(t)\right\|}
$$

Denote $\mathbf{n}_{\theta_{R O \perp}}$ as the unit vector perpendicular to $\mathbf{n}_{R O}$, and $\theta_{R O}-\theta_{R O \perp}=\pi / 2$, where $\theta_{R O \perp}$ is the argument of unit vector $\mathbf{n}_{\theta_{R O \perp}}$. Thus we have

$$
\nabla_{\mathbf{P}} \theta_{R O}=\frac{\mathbf{n}_{\theta_{R O \perp}}}{P_{d}\left(\mathbf{P}, \mathbf{P}_{o b s}\right)}
$$

Therefore

$$
\nabla_{\mathbf{P}} \theta_{d}=\nabla_{\mathbf{P}}\left\|\theta-\theta_{R O}\right\|
$$

If $\theta>\theta_{R O}$, we obtain

$$
\nabla_{\mathbf{P}} \theta_{d}=\nabla_{\mathbf{P}}\left(\theta-\theta_{R O}\right)=\frac{-\mathbf{n}_{\theta_{R O \perp}}}{P_{d}\left(\mathbf{P}, \mathbf{P}_{o b s}\right)}
$$

If $\theta \leq \theta_{R O}$, we have

$$
\nabla_{\mathbf{P}} \theta_{d}=\nabla_{\mathbf{P}}\left(\theta_{R O}-\theta\right)=\frac{\mathbf{n}_{\theta_{R O \perp}}}{P_{d}\left(\mathbf{P}, \mathbf{P}_{o b s}\right)}
$$

However one can notice that when $\theta>\theta_{R O}, \mathbf{n}_{\theta_{R O \perp}}=$ $-\mathbf{n}_{R O \perp}$, and when $\theta \leq \theta_{R O}, \mathbf{n}_{\theta_{R O \perp}}=\mathbf{n}_{R O \perp}$, thus one can obtain that

$$
\nabla_{\mathbf{P}} \theta_{d}=\frac{\mathbf{n}_{R O \perp}}{P_{d}\left(\mathbf{P}, \mathbf{P}_{o b s}\right)}
$$

Therefore we have

$$
\begin{aligned}
& \mathbf{S}_{r e p}(\mathbf{P}, \mathbf{v})= \\
& \left\{\begin{array}{l}
0, \text { if } P_{d}\left(\mathbf{P}, \mathbf{P}_{o b s}\right)-P_{m} \geq p_{0} \text { or } V_{R O} \leq 0 \\
\mathbf{S}_{r e p 1}+\mathbf{S}_{r e p 2}, \\
\quad \text { if } 0<P_{d}\left(\mathbf{P}, \mathbf{P}_{o b s}\right)-P_{m}<p_{0} \text { and } V_{R O}>0
\end{array}\right.
\end{aligned}
$$

where

$$
\mathbf{S}_{r e p 1}=\frac{-K_{p v} \cos \theta_{d}}{\left(P_{d}\left(\mathbf{P}, \mathbf{P}_{o b s}\right)-P_{m}\right)^{2}}\left(1+\frac{V_{R O}}{a_{\max }}\right) \mathbf{n}_{R O}
$$

and

$$
\begin{aligned}
\mathbf{S}_{r e p 2} & =\frac{K_{p v} V_{R O} V_{R O \perp} \cos \theta_{d}}{a_{m a x} P_{d}\left(\mathbf{P}, \mathbf{P}_{o b s}\right)\left(P_{d}\left(\mathbf{P}, \mathbf{P}_{o b s}\right)-P_{m}\right)^{2}} \mathbf{n}_{R O \perp} \\
& +K_{p v} \sin \theta_{d}\left(\frac{1}{P_{d}\left(\mathbf{P}, \mathbf{P}_{o b s}\right)-P_{m}}-\frac{1}{p_{0}}\right) \frac{\mathbf{n}_{R O \perp}}{P_{d}\left(\mathbf{P}, \mathbf{P}_{o b s}\right)}
\end{aligned}
$$

We also have

$$
\begin{aligned}
& \mathbf{S}_{r e p}(\theta, \omega)= \\
& \left\{\begin{array}{l}
0, \text { if } V_{R O}<0 \text { or } P_{d}\left(\mathbf{P}, \mathbf{P}_{o b s}\right)>p_{\theta} \text { or } \theta_{d}>\theta_{0} \\
\mathbf{S}_{r e p 3}+\mathbf{S}_{r e p 4} \\
\text { if } V_{R O}>0 \text { and } P_{d}\left(\mathbf{P}, \mathbf{P}_{o b s}\right) \leq p_{\theta} \text { and } \theta(\omega)<\theta_{d} \leq \theta_{0} \\
\mathbf{S}_{r e p 5}+\mathbf{S}_{r e p 6}, \\
\text { if } V_{R O}>0 \text { and } P_{d}\left(\mathbf{P}, \mathbf{P}_{o b s}\right)<p_{\theta} \text { and } \theta_{d}<\theta(\omega)
\end{array}\right.
\end{aligned}
$$

where

$$
\begin{gathered}
\mathbf{S}_{r e p 3}=-2 M^{2} H\left(\theta_{0}-\theta_{d}\right)^{2} \mathbf{n}_{R O} \\
\mathbf{S}_{r e p 4}=4 K_{\theta 1} M H^{2}\left(\theta_{d}-\theta(\omega)\right)\left(\theta_{0}-\theta_{d}\right)^{2} \frac{\mathbf{n}_{R O \perp}}{P_{d}\left(\mathbf{P}, \mathbf{P}_{o b s}\right)} \\
+2 M^{2} H^{2}\left(\theta_{0}-\theta_{d}\right) \frac{\mathbf{n}_{R O \perp}}{P_{d}\left(\mathbf{P}, \mathbf{P}_{o b s}\right)}
\end{gathered}
$$

and

$$
\begin{gathered}
\mathbf{S}_{r e p 5}=-2 K_{\theta 2}^{2} H\left(\theta_{0}-\theta_{d}\right)^{2} \mathbf{n}_{R O} \\
\mathbf{S}_{r e p 6}=2 K_{\theta 2}^{2} H^{2}\left(\theta_{0}-\theta_{d}\right) \frac{\mathbf{n}_{R O \perp}}{P_{d}\left(\mathbf{P}, \mathbf{P}_{o b s}\right)}
\end{gathered}
$$

where $M=-K_{\theta 1}\left(\theta_{d}-\theta(\omega)\right)^{2}+K_{\theta 2}$ and $H=p_{\theta}-P_{d}\left(\mathbf{P}, \mathbf{P}_{o b s}\right)$.

The relationship among the repulsive reference velocity components is shown in Fig. 5. The repulsive forces $\mathbf{S}_{r e p 1}$, $\mathbf{S}_{r e p 3}$ and $\mathbf{S}_{r e p 5}$ are in the opposite direction of $\mathbf{n}_{R O}$, which keep the robot away from obstacles. The repulsive forces $\mathbf{S}_{r e p 2}, \mathbf{S}_{r e p 4}$ and $\mathbf{S}_{r e p 6}$ are in the direction of $\mathbf{n}_{R O \perp}$, which drive the robot for detouring. One can see that because of the new repulsive function $U_{r e p}(\theta, \omega)$, another force is generated 


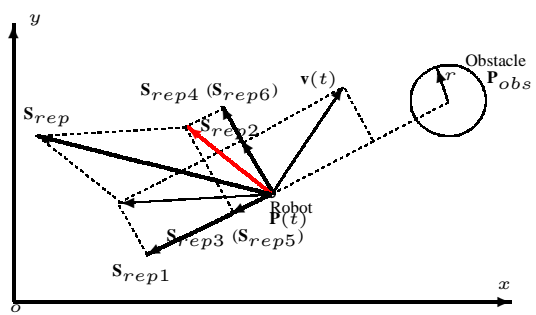

Fig. 5. Relationship among $\mathbf{S}_{r e p 1}, \mathbf{S}_{r e p 2}, \mathbf{S}_{r e p 3}\left(\mathbf{S}_{r e p 5}\right)$ and $\mathbf{S}_{r e p 4}$ $\left(\mathbf{S}_{r e p 6}\right)$

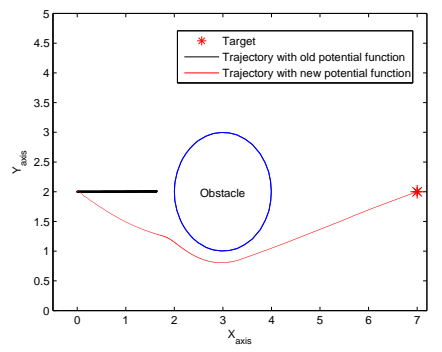

Fig. 6. Situation with the connection between the robot and the target and the robot velocity pass through the center of the obstacle

to repulse the robot away from the obstacle, shown as the red arrow in Fig. 5. When there are multiple obstacles, the repulsive force can be calculated by

$$
\mathbf{S}_{r e p}=\sum_{j=1}^{n_{\text {obs }}} \mathbf{S}_{r e p, j}
$$

where $n_{\text {obs }}$ is the number of obstacles in the sensor, and $\mathbf{S}_{r e p, j}$ is the repulsive force generated by the $j^{\text {th }}$ obstacle. The total force can be obtained by

$$
\mathbf{S}_{\text {total }}=\mathbf{S}_{a t t}+\mathbf{S}_{r e p}
$$

and the total force $\mathbf{S}_{t o t a l}$ is used for motion planning.

\section{Simulation RESUlts}

In the simulation, parameters are set as follows: maximum linear acceleration $a_{\max }=2.0 \mathrm{~m} / \mathrm{s}^{2}$, maximum angular acceleration $\beta_{\max }=1.0 \mathrm{rad} / \mathrm{s}$, obstacle influence range for $U_{r e p}(\mathbf{P}, \mathbf{v}): p_{0}=0.3 \mathrm{~m}$, obstacle influence range for $U_{\text {rep }}(\theta, \omega): p_{\theta}=0.6 \mathrm{~m}$, time window $T=3 \mathrm{~s}$, influence angle $\theta_{0}=\pi / 4 . K_{p}=50, K_{I}=100, K_{a t t}=0.04$, $K_{p v}=0.8$ and $K_{\theta 1}=K_{\theta 2}=0.8$,

$$
\alpha(x, y, \dot{x}, \dot{y})=\left[\begin{array}{ll}
\operatorname{sgn}(\cos \theta) & -\operatorname{sgn}(\sin \theta) \\
\operatorname{sgn}(\sin \theta) & \operatorname{sgn}(\cos \theta)
\end{array}\right],
$$

where $\operatorname{sgn}(\sigma)$ is the sign function which extracts the sign.

The first simulation is made in which the connection between the robot and the target and the robot velocity pass through the center of the obstacle. As we can see in Fig. 6 , with the classical potential function defined in [11], the robot stops in front of the obstacle. However with our new potential function, the robot manages to avoid the obstacle and arrive the target.
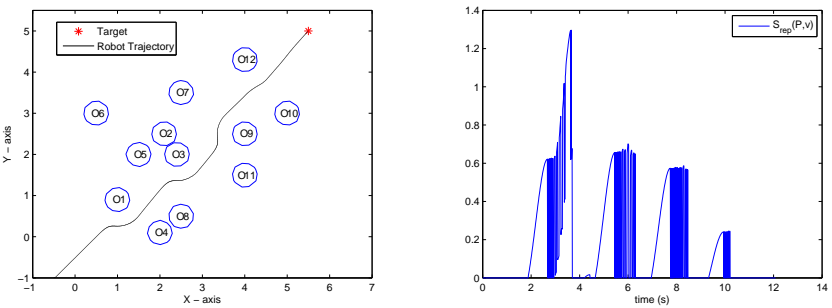

Fig. 7. Robot trajectory with Fig. 8. Repulsive force of original original $\mathbf{S}_{r e p}(\mathbf{P}, \mathbf{v}) \quad \mathbf{U}_{r e p}(\mathbf{P}, \mathbf{v})$
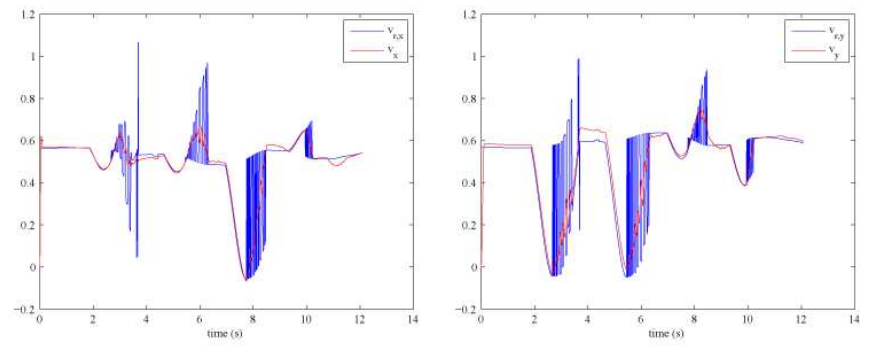

Fig. 9. Velocity tracking in $\mathrm{X}$ and $\mathrm{Y}$ direction with original $\mathbf{S}_{r e p}(\mathbf{P}, \mathbf{v})$

The second simulation is made using the original repulsive function of [11] in comparison with our new repulsive function. Although the robot is able to avoid obstacles (Fig. 7), there are many unexpected oscillations in the generated repulsive force (Fig. 8), which eventually result in oscillations in the generated references (Fig. 9), this is detrimental for velocity tracking. And one can see that there are also many oscillations in the generated controls (Fig. 10).

The last simulation is shown from Fig. 11 to Fig. 14, where both new repulsive forces $\mathbf{S}_{r e p}(\theta, \omega)$ and $\mathbf{S}_{r e p}(\mathbf{P}, \mathbf{v})$ are imposed on the robot to avoid obstacles. The robot trajectory is shown in Fig. 11, the forces generated by the potential field function are shown in Fig. 12, and we can see that the forces change smoothly without sudden changes. Velocity tracking results are shown in Fig. 13, it is clear that reference velocities also change smoothly, the controller is able to track the velocity references. The control inputs calculated by the $i$-PID controller are shown in Fig. 14. One can see that with both repulsive forces $\mathbf{S}_{r e p}(\theta, \omega)$ and $\mathbf{S}_{r e p}(\mathbf{P}, \mathbf{v})$, the robot is
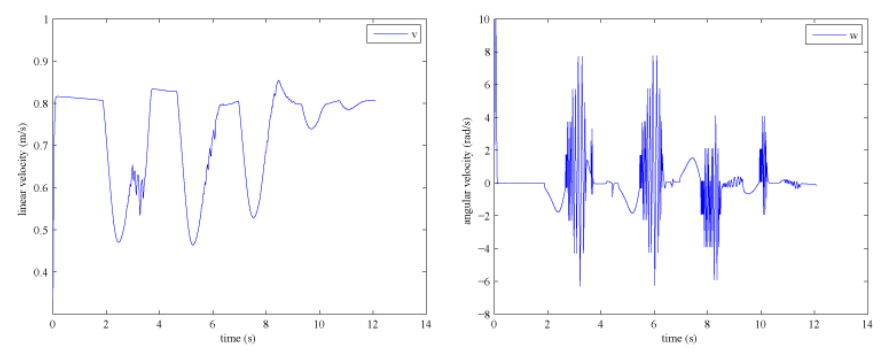

Fig. 10. Linear velocity control and angular velocity control with original $\mathbf{S}_{r e p}(\mathbf{P}, \mathbf{v})$ 


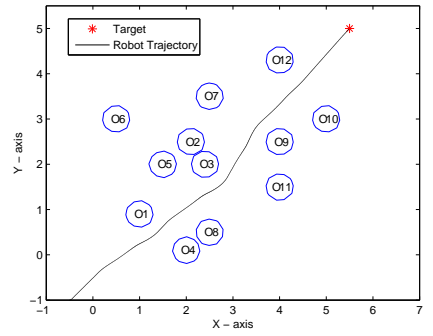

Fig. 11. Robot trajectory with both $\mathbf{S}_{r e p}(\theta, \omega)$ and $\mathbf{S}_{r e p}(\mathbf{P}, \mathbf{v})$
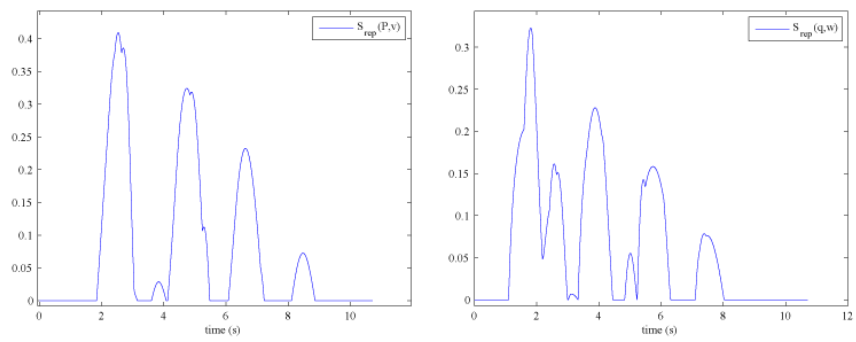

Fig. 12. Repulsive force $\mathbf{S}_{r e p}(\mathbf{P}, \mathbf{v})$ and $\mathbf{S}_{r e p}(\theta, \omega)$ with both $\mathbf{S}_{r e p}(\theta, \omega)$ and $\mathbf{S}_{r e p}(\mathbf{P}, \mathbf{v})$

able to avoid all the obstacles and keep a predefined distance from obstacles, and there is no oscillations in repulsive forces and input controls.

A $3 D$ simulation made by using ROS (Robot Operating System) and an implementation in a real robot are in the attached video.

\section{CONCLUSION}

This paper proposes a motion planning approach for nonholonomic mobile robots using the $i$-PID controller and a new potential field method. The $i$-PID controller is determined to track the desired velocity, and the desired velocity is obtained from the new potential field function. The new potential field function, which takes into account the robot orientation and angular velocity, improves the performance of obstacle avoidance and can produce smooth repulsive force to avoid oscillations in complex environment.
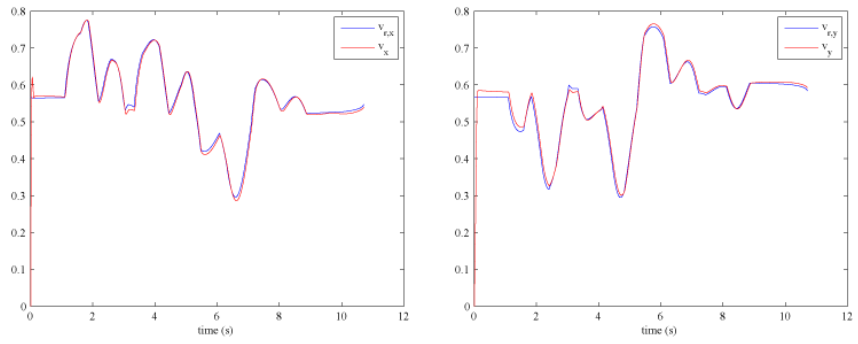

Fig. 13. Velocity tracking in $\mathrm{X}$ and $\mathrm{Y}$ direction with both $\mathbf{S}_{r e p}(\theta, \omega)$ and $\mathbf{S}_{r e p}(\mathbf{P}, \mathbf{v})$
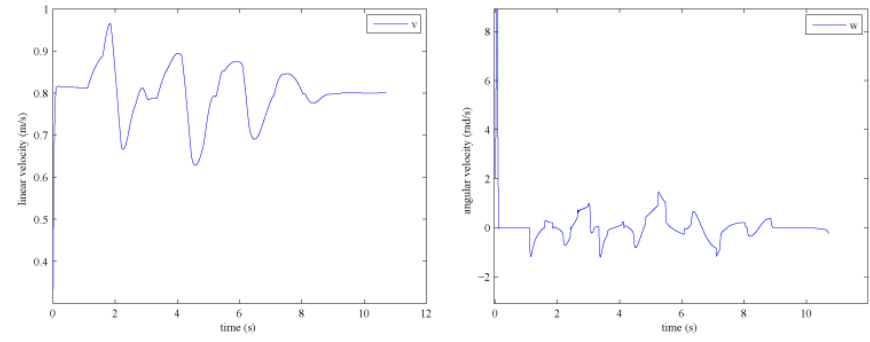

Fig. 14. Linear velocity control and angular velocity control with both $\mathbf{S}_{r e p}(\theta, \omega)$ and $\mathbf{S}_{r e p}(\mathbf{P}, \mathbf{v})$

\section{REFERENCES}

[1] S. LaValle, Planning Algorithms. Cambridge University Press, 2006.

[2] N. J. Nilsson, Principles of Artificial Intelligence. Tioga Publishing Company, 1980.

[3] A. Stentz, "Optimal and efficient path planning for partially-known environments," in Robotics and Automation, 1994. Proceedings., 1994 IEEE International Conference on. IEEE, 1994, pp. 3310-3317.

[4] Y. Bin-qiang, Z. Ming-fu, and W. Yi, "Research of path planning method for mobile robot based on artificial potential field," in Multimedia Technology, International Conference on, 2011, pp. 3192-3195.

[5] D. Bilò, Y. Disser, M. Mihalák, S. Suri, E. Vicari, and P. Widmayer, "Reconstructing visibility graphs with simple robots," Theoretical Computer Science, vol. 444, pp. 52-59, 2012.

[6] M. Defoort, J. Palos, A. Kokosy, T. Floquet, and W. Perruquetti, "Performance-based reactive navigation for non-holonomic mobile robots," Robotica, vol. 27, no. 02, pp. 281-290, 2009.

[7] O. Khatib, "Real-time obstacle avoidance for manipulators and mobile robots," The international journal of robotics research, vol. 5, no. 1, pp. 90-98, 1986.

[8] J.-C. Latombe, ROBOT MOTION PLANNING. Springer, 1991.

[9] H. Rezaee and F. Abdollahi, "Adaptive artificial potential field approach for obstacle avoidance of unmanned aircrafts," in Advanced Intelligent Mechatronics, 2012 IEEE/ASME International Conference on, 2012, pp. 1-6.

[10] R. Conn and M. Kam, "Robot motion planning on n-dimensional star worlds among moving obstacles," Robotics and Automation, IEEE Transactions on, vol. 14, no. 2, pp. 320-325, 1998.

[11] S. Ge and Y. Cui, "Dynamic motion planning for mobile robots using potential field method," Autonomous Robots, vol. 13, no. 3, pp. 207222, 2002.

[12] S. S. Ge and Y. J. Cui, "New potential functions for mobile robot path planning," Robotics and Automation, IEEE Transactions on, vol. 16, no. 5, pp. 615-620, 2000.

[13] J. Reeds and L. Shepp, "Optimal paths for a car that goes both forwards and backwards," Pacific Journal of Mathematics, vol. 145, no. 2, pp. 367-393, 1990.

[14] R. W. Brockett, Asymptotic stability and feedback stabilization. Defense Technical Information Center, 1983.

[15] C. Samson and K. Ait-Abderrahim, "Feedback control of a nonholonomic wheeled cart in cartesian space," in IEEE International Conference on Robotics and Automation, 1991, pp. 1136-1141.

[16] A. De Luca and G. Oriolo, "Local incremental planning for nonholonomic mobile robots," in Robotics and Automation. IEEE International Conference on, 1994, pp. 104-110 vol.1.

[17] M. Fliess and C. Join, "Model-free control and intelligent PID controllers: towards a possible trivialization of nonlinear control?" in 15th IFAC Symposium on System Identification, Saint-Malo, France, 2009.

[18] M. Fliess, C. Join, and S. Riachy, "Revisiting some practical issues in the implementation of model-free control," in 18th IFAC World Congress, IFAC WC'2011, Aug. 2011.

[19] Y. Ma, G. Zheng, W. Perruquetti, and Z. Qiu, "Control of nonholonomic wheeled mobile robots via i-pid controller," in Intelligent Robots and Systems (IROS), 2013 IEEE/RSJ International Conference on, Nov 2013, pp. 4413-4418. 